\title{
The effectiveness and value of nadofaragene firadenovec, oportuzumab monatox, and pembrolizumab for BCG-unresponsive non-muscle-invasive bladder cancer
}

\author{
A summary from the Institute for Clinical and Economic Review's Midwest Comparative \\ Effectiveness Public Advisory Council
}

Molly Beinfeld, MPH; Steven J Atlas, MD, MPH; Daniel Touchette, PharmD, MA; Avery McKenna; David Rind, MD; and Steven D Pearson, MD, MSc

Bladder cancer is the sixth most common cancer in the United States. Approximately 80,000 new cases are diagnosed each year, and more than 17,000 people die from the disease annually. ${ }^{1,2}$ At diagnosis, most patients have non-muscle-invasive bladder cancer (NMIBC), meaning that the cancer involves the inner lining of the bladder but has not invaded into the deeper muscle layer. NMIBC includes 3 subgroups that are associated with increasing risk of cancer progression: (1) $70 \%$ of patients have Ta disease, with polyps extending into the lining of the bladder; (2) $20 \%$ of patients have T1 disease, with tumors below the superficial lining but not involving the muscular layer of the bladder wall; and (3) 10\% of patients have carcinoma in situ (CIS) with flat, superficial growths. ${ }^{3}$ Treatment and related health care costs for patients with bladder cancer is estimated to be $\$ 4-\$ 5$ billion annually in the United States. ${ }^{4}$

Treatment of NMIBC involves removal of visible cancer followed by intravesical therapy for those at increased risk of progression to invasive disease. The most common initial intravesical therapy is Bacillus
Calmette-Guerin (BCG), an attenuated live form of Mycobacterium bovis. ${ }^{5}$ For patients with invasive disease or who are not responding to or tolerating intravesical therapy, cystectomy may be performed to remove the bladder.,7

Although short-term prognosis for NMIBC is good, patients with BCG-unresponsive disease can select cystectomy or further intravesical chemotherapy with agents such as gemcitabine monotherapy or combination gemcitabine plus docetaxel. Response rates to these agents are relatively low, underscoring the need for new bladder-sparing treatment options. ${ }^{8,9}$

In January 2020, pembrolizumab (Keytruda), a systemically administered immunotherapy agent, received an additional indication for BCG-unresponsive CIS disease. ${ }^{10}$ In addition, 2 new intravesical therapies have recently begun the US Food and Drug Administration (FDA) approval process: nadofaragene firadenovec (Adstiladrin) and oportuzumab monatox (Vicineum). Nadofaragene firadenovec uses a recombinant adenovirus vector that encodes the human interferon alfa-2b gene with a surfactant to enhance transfer into

\author{
Author affiliations \\ Molly Beinfeld, MPH; Avery McKenna; \\ David Rind, MD; and Steven D Pearson, \\ MD, MSc, Institute for Clinical and \\ Economic Review, Boston, MA. Steven \\ J Atlas, MD, MPH, Division of General \\ Internal Medicine, Massachusetts General \\ Hospital and Harvard Medical School, \\ Boston, MA. Daniel Touchette, PharmD, \\ MA, University of Illinois at Chicago \\ College of Pharmacy, Chicago, IL. \\ AUTHOR CORRESPONDENCE: \\ Molly Beinfeld, mbeinfeld@icer.org \\ J Manag Care Spec Pharm. \\ 2021;27(6):797-804 \\ Copyright $\odot 2021$, Academy of Managed \\ Care Pharmacy. All rights reserved.
}

cancer cells. ${ }^{11,12}$ It is instilled every 3 months. Oportuzumab monatox is a recombinant fusion protein with a cell adhesion molecule antibody linked to a toxin that binds to the cancer cell that releases the toxin into the cell, inducing cell death. ${ }^{13}$ It is instilled twice a week for 6 weeks, then weekly 


\section{FIGURE 1 Phase 3 Results of Nadofaragene Firadenovec: Complete Response and High-Grade Recurrence Free Survival, $\mathrm{CIS} \pm \mathrm{Ta} / \mathrm{T} 1$, and $\mathrm{Ta} / \mathrm{T}^{14}$}

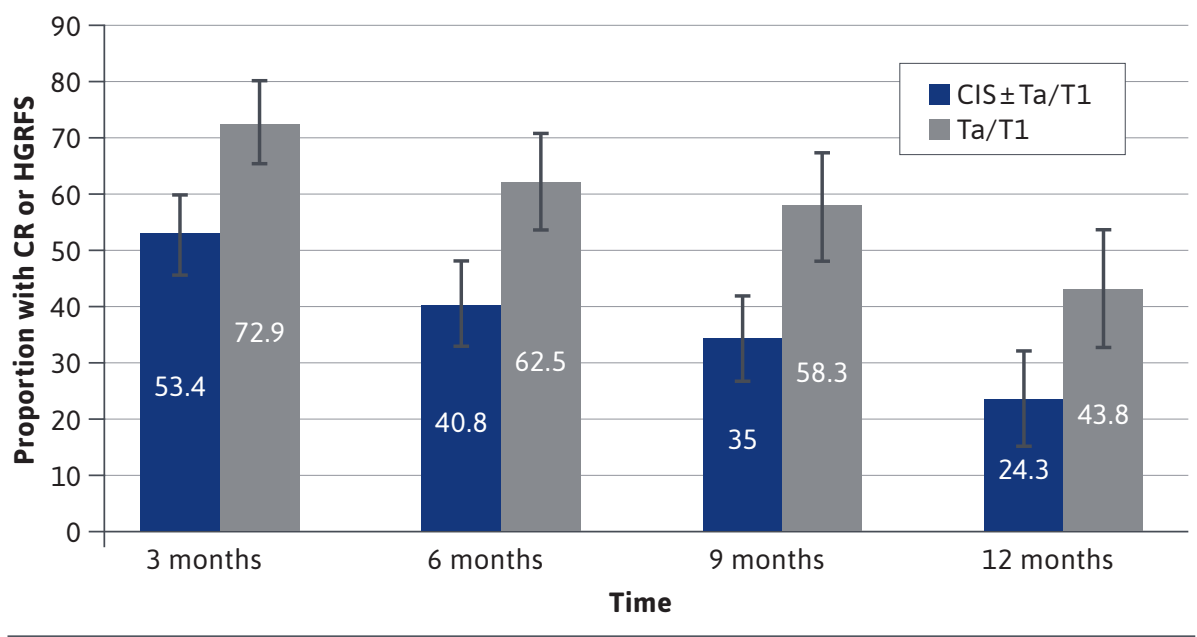

Note: Error bars represent standard error of proportion with CR or HGRFS.

$C I S=$ carcinoma in situ; $C R=$ complete response; $H G R F S=$ high-grade recurrence free survival; Ta disease=polyps extending into the lining of the bladder; T1 disease=tumors below the superficial lining but not involving the muscular layer of the bladder wall.

\section{FIGURE 2 Phase 3 Results of Oportuzumab Monatox: Complete Response and High-Grade Recurrence Free Survival, $\mathrm{CIS} \pm \mathrm{Ta} / \mathrm{T} 1$, and $\mathrm{Ta} / \mathrm{T}^{\mathrm{a}}$}

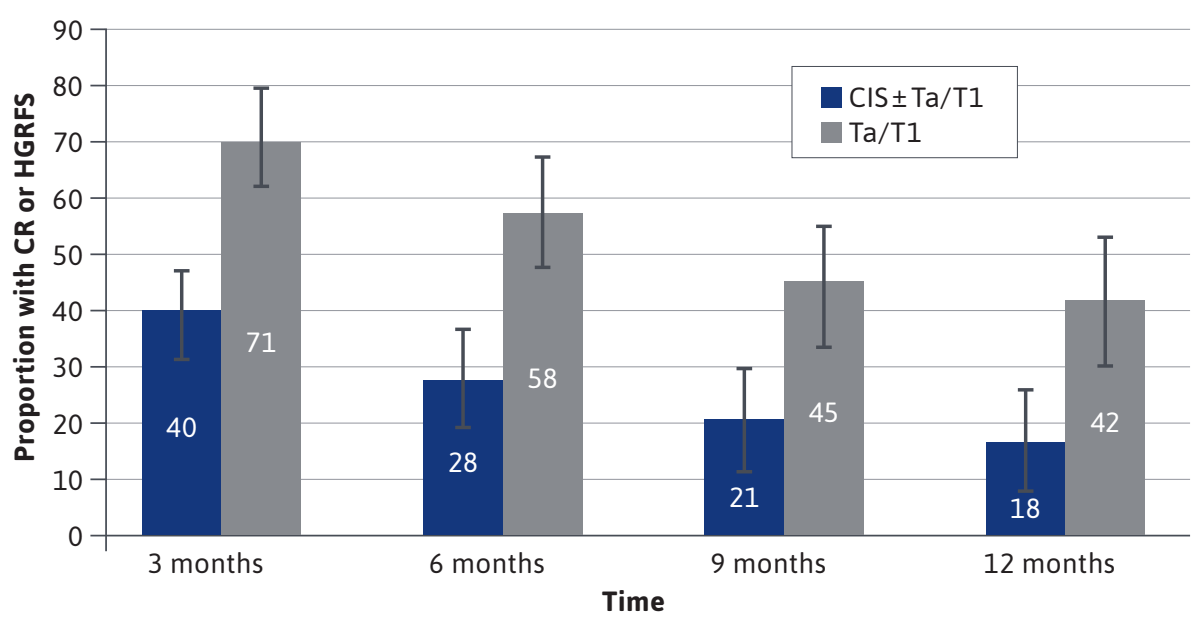

Note: Error bars represent standard error of proportion with CR or HGRFS.

aUnpublished data, Sesen Bio, 2020.

$C I S=$ carcinoma in situ; $C R=$ complete response; $H G R F S=$ high-grade recurrence free survival;

Ta disease = polyps extending into the lining of the bladder; TI disease=tumors below the superficial lining but not involving the muscular layer of the bladder wall. for 6 weeks and then every 2 weeks for up to 2 years.

The Institute for Clinical and Economic Review (ICER) conducted a systematic literature review and cost-effectiveness analysis to evaluate the health and economic outcomes of nadofaragene firadenovec, oportuzumab monatox, and pembrolizumab for BCG-unresponsive NMIBC. Complete details of ICER's systematic literature search and protocol, as well as the methodology and model structure for the economic evaluation are available on ICER's website at https://icer.org/assessment/ bladder-cancer-2020/.

In this report, we present the summary of our findings and highlights of the policy discussion with key stakeholders held at a public meeting of the Midwest Comparative Effectiveness Public Advisory Council (CEPAC) on November 20, 2020.

\section{Summary of Findings}

\section{CLINICAL EFFECTIVENESS}

The systematic literature review identified only one phase 3 singlearm, open-label prospective study for nadofaragene and oportuzumab monatox and one phase 2 study of pembrolizumab, making indirect comparisons of relative effectiveness difficult. At the time of the review, only the pivotal trial of nadofaragene firadenovec had been published ${ }^{14}$ and our review also evaluated information from conference abstracts, regulatory documents, and data submitted by the manufacturers.

The pivotal trials of nadofaragene firadenovec and oportuzumab monatox included similar distributions of patients with CIS $\pm \mathrm{Ta} / \mathrm{T} 1$ and non-CIS with high-grade Ta/T1 disease and used the same definitions of BCGunresponsive disease. However, the nadofaragene firadenovec pivotal trial 


\section{FIGURE 3 Phase 2 Results of Pembrolizumab: Complete Response and $\mathrm{CIS} \pm \mathrm{Ta} / \mathrm{T} 1^{16}$}

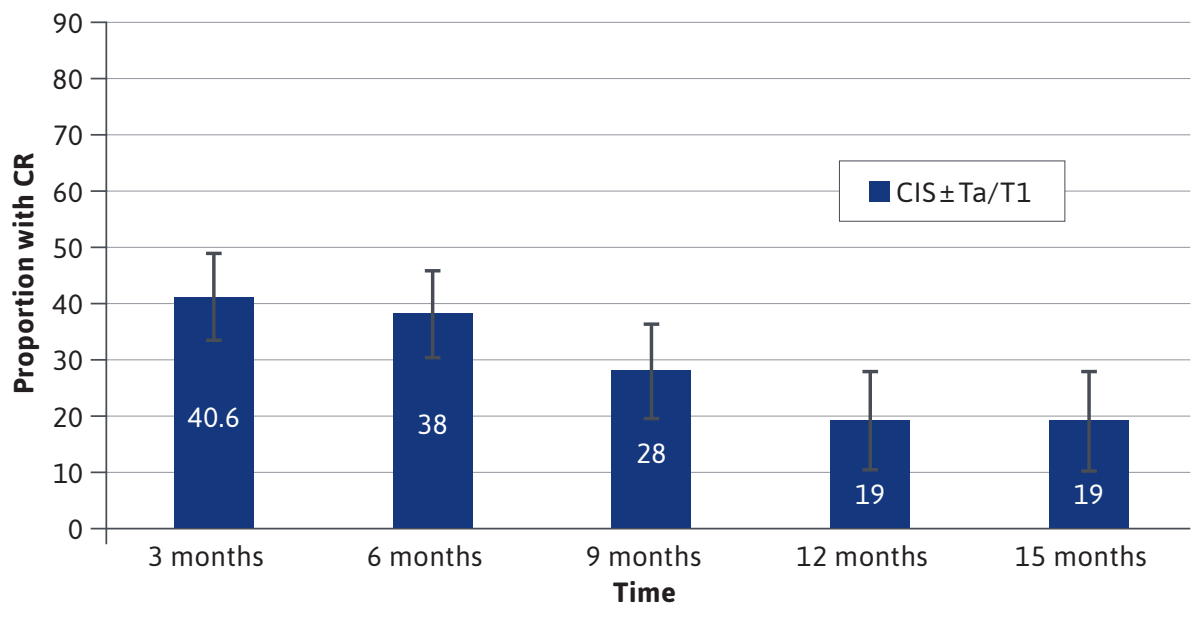

Note: Error bars represent standard error of proportion with $C R$.

$\mathrm{Cl}=$ carcinoma in situ; $\mathrm{CR}=$ complete response; $T$ a disease=polyps extending into the lining of the bladder; T1 disease =tumors below the superficial lining but not involving the muscular layer of the bladder wall.

required a biopsy at the 12 -month evaluation, whereas the oportuzumab monatox pivotal trial did not. This biopsy could have resulted in additional patients being identified as having recurrent disease who would not have been found without a biopsy. Efficacy outcomes were reported for all eligible patients in the nadofaragene firadenovec trial who received the study drug, whereas patients who did not complete induction therapy were excluded in the oportuzumab monatox trial. At the time of this publication, only data from the CIS $\pm \mathrm{Ta}$ / T1 cohort of the pivotal trial of pembrolizumab were available. This trial included the additional inclusion criteria that patients either be ineligible for or decline cystectomy. The most common outcomes reported in the pivotal trials were complete response (CR) and high-grade recurrence free survival (HGRFS) at prespecified time points after initial evaluation (unpublished data, Sesen Bio, 2020; Merck, 2020). ${ }^{14}$
In the pivotal trial of nadofaragene firadenovec, 55 (53\%) of 103 patients with $\mathrm{CIS} \pm \mathrm{Ta} / \mathrm{T} 1$ disease achieved a $\mathrm{CR}$ at 3 months, declining to $24 \%$ at 12 months. For patients with highgrade $\mathrm{Ta}$ /T1 disease alone, HGRFS was 35 (73\%) of 48 patients at 3 months and $44 \%$ at 12 months (Figure 1). ${ }^{14,15}$

In the pivotal trial of oportuzumab monatox, 36 (40\%) of 89 patients with $\mathrm{CIS} \pm \mathrm{Ta} / \mathrm{T} 1$ disease achieved a $\mathrm{CR}$ at 3 months, declining to $17 \%$ at 12 months. For patients with highgrade $\mathrm{Ta}$ /T1 disease alone, HGRFS was 27 (71\%) of 38 patients at 3 months and $42 \%$ at 12 months (Figure 2; unpublished data, Sesen Bio, 2020).

In the pivotal trial of pembrolizumab among patients with CIS $\pm \mathrm{Ta}$ / T1 disease, 39 (41\%) of 96 patients achieved a $\mathrm{CR}$ at 3 months, which declined to $19 \%$ at 12 months (Figure 3). Outcomes for the Ta/T1 cohort were not available at the time of the report (unpublished data, Merck, 2020). ${ }^{16}$

Harms recorded in these trials included treatment-emergent adverse events (TEAEs) and discontinuation. For the 2 intravesical therapies, TEAEs generally included bladder-related symptoms such as irritative voiding, hematuria, and urinary tract infection. For pembrolizumab, TEAEs included immune-mediated adverse events (AEs) and infusion reactions. In the pivotal trial of nadofaragene firadenovec, 146 (93\%) of 157 patients evaluated for safety reported at least 1 TEAE, of which 14 (9\%) were serious, but only 3 (2\%) patients discontinued due to TEAEs. ${ }^{14}$ In the pivotal trial of oportuzumab monatox, 117 (88\%) of 133 patients in the safety population reported any TEAE, of which 19 (14\%) were serious, with 4 (3\%) patients discontinuing treatment due to TEAEs (unpublished data, Sesen Bio, 2020). In the pivotal trial of pembrolizumab, 99 (97\%) of 102 patients in the safety population reported any $\mathrm{AE}$, of which 26 (26\%) were serious, with 10 (10\%) patients discontinuing due to AEs (unpublished data, Merck, 2020). ${ }^{16}$

\section{LIMITATIONS OF THE CLINICAL EVIDENCE}

The primary limitation in the clinical evidence for these 3 agents is the lack of data from randomized controlled trials. The FDA permitted single arm trials for this population because randomizing patients to placebo or minimally effective therapies was deemed unethical. The lack of head-to-head comparative data near the time of launch is not unexpected, but the lack of randomization in the pivotal trials makes even indirect comparisons needed to guide judgments of relative effectiveness highly uncertain.

Differences in study populations between the single arm trials and in assessing and reporting outcomes further complicate the comparability of the trial results. Although outcomes of nadofaragene firadenovec and oportuzumab monatox show 


\section{TABLE 1}

\section{Incremental Cost-Effectiveness Ratios Compared With Hypothetical Treatment Comparator in Patients With $\mathrm{CIS} \pm \mathrm{Ta} / \mathrm{T} 1$}

\begin{tabular}{l|c|c|c}
\hline \multicolumn{1}{c|}{ Intervention } & $\begin{array}{c}\text { Cost per QALY } \\
\text { gained }\end{array}$ & Cost per evLYG & $\begin{array}{c}\text { Cost per year in } \\
\text { progression-free } \\
\text { state }\end{array}$ \\
\hline Nadofaragene firadenovec & $\$ 151,000$ & $\$ 135,000$ & $\$ 100,000$ \\
\hline Oportuzumab monatox & $\$ 382,000$ & $\$ 343,000$ & $\$ 281,000$ \\
\hline Pembrolizumab & $\$ 114,000$ & $\$ 103,000$ & $\$ 76,000$ \\
\hline
\end{tabular}

$C I S=$ carcinoma in situ; evLYG = equal value life-year gained; $Q A L Y=$ quality-adjusted life-year;

Ta disease = polyps extending into the lining of the bladder; $T 1$ disease = tumors below the superficial lining but not involving the muscular layer of the bladder wall.

\section{TABLE 2}

\section{Incremental Cost-Effectiveness Ratios Compared With Hypothetical Treatment Comparator in Patients With High-Grade Ta/T1 Disease Alone}

\begin{tabular}{|c|c|c|c|}
\hline Intervention & $\begin{array}{c}\text { Cost per QALY } \\
\text { gained }\end{array}$ & Cost per evLYG & $\begin{array}{c}\text { Cost per year } \\
\text { in progression-free } \\
\text { state }\end{array}$ \\
\hline Nadofaragene firadenovec & $\$ 93,000$ & $\$ 87,000$ & $\$ 65,000$ \\
\hline Oportuzumab monatox & $\$ 123,000$ & $\$ 117,000$ & $\$ 88,000$ \\
\hline
\end{tabular}

evLYG = equal value life-year gained; $Q A L Y=$ quality-adjusted life-year; Ta disease = polyps extending into the lining of the bladder; T1 disease=tumors below the superficial lining but not involving the muscular layer of the bladder wall response rates that are similar to or better than currently available treatments, such as gemcitabine with or without docetaxel, there continues to be considerable uncertainty about their long-term efficacy. Because they are administered in the bladder, they appear to have few serious side effects. For pembrolizumab, the trial data suggest similar response rates, but because it is given systemically, more patients report side effects, and its use is associated with infrequent but potentially serious AEs.

\section{LONG-TERM COST-EFFECTIVENESS}

Lacking comparative data on which to base our incremental cost-effectiveness analysis, we evaluated all agents compared with a hypothetical comparator treatment whose 3-month CR rate could be varied in a sensitivity analysis. For the base case, this hypothetical treatment was completely ineffective, with a $\mathrm{CR}$ of $0 \%$ at 3 months. We evaluated the cost-effectiveness of all treatments in 2 populations: $\mathrm{CIS} \pm \mathrm{Ta}$ /T1 (population 1) and high-grade Ta/T1 disease alone (population 2). We developed a de novo semi-Markov decision analytic model with model structure and inputs informed by key clinical trials, previous economic models, systematic literature reviews, and input from stakeholders (patients, advocacy groups, clinicians, payers, researchers, and manufacturers).

Costs and utilities for model health states were derived from 3 key studies. ${ }^{17-19}$ Since the prices for nadofaragene firadenovec and oportuzumab monatox were not available at the time of the report, the price for nadofaragene firadenovec was set to equal the annual price of pembrolizumab $(\$ 164,337)$, whereas oportuzumab was set at $\$ 150,000$ per year net of all rebates based on analyst estimates. Full details on ICER's cost-effectiveness analysis and model are available on ICER's website at https://icer.org/assessment/ bladder-cancer-2020.

For each population, we estimated time in progression-free state, total costs, total quality-adjusted life-years (QALYs), total equal value life-years gained (evLYGs), and total life-years (LYs) for each treatment over a lifetime time horizon. Base-case analyses were assessed from the health care perspective. A discount rate of $3 \%$ was applied to all costs, QALYs, evLYGs, and LYs.

The base-case cost-effectiveness, with each drug compared with the hypothetical comparator with a $0 \%$ CR at 3 months, were $\$ 151,000$ per QALY gained for nadofaragene firadenovec, \$382,000 per QALY gained for oportuzumab monatox, and $\$ 114,000$ per QALY gained for pembrolizumab. Tables 1 and 2 summarize the full results for all incremental costeffectiveness outcomes evaluated. In scenario analyses, when the CR of the hypothetical comparator was increased to $20 \%$ in the CIS $\pm \mathrm{Ta} / \mathrm{T} 1$ population, the incremental costeffectiveness ratio for nadofaragene firadenovec and oportuzumab monatox increased to $\$ 155,000$ and $\$ 407,000$, respectively. We did not conduct scenario analyses for pembrolizumab. In the high-grade $\mathrm{Ta} / \mathrm{T} 1$ population, altering the hypothetical comparator's CR to $30 \%$ resulted in incremental cost-effectiveness ratios of $\$ 107,000$ for nadofaragene firadenovec and $\$ 147,000$ for oportuzumab monatox. 


\section{TABLE 3 Votes on Other Benefits and Contextual Considerations for Nadofaragene Firadenovec and Oportuzumab Monatox}

\begin{tabular}{|c|c|c|}
\hline 1 (suggests lower value) & 2 (intermediate) & 3 (suggests higher value) \\
\hline $\begin{array}{l}\text { This intervention will not differentially benefit a } \\
\text { historically disadvantaged or underserved community }\end{array}$ & \multirow[b]{2}{*}{6 votes } & $\begin{array}{l}\text { This intervention will differentially benefit a historically } \\
\text { disadvantaged or underserved community }\end{array}$ \\
\hline 5 votes & & 0 votes \\
\hline 1 (suggests lower value) & 2 (intermediate) & 3 (suggests higher value) \\
\hline $\begin{array}{l}\text { Small health loss without this treatment as measured by } \\
\text { absolute QALY shortfall }\end{array}$ & \multirow[b]{2}{*}{4 votes } & $\begin{array}{l}\text { Substantial health loss without this treatment as } \\
\text { measured by absolute QALY shortfall }\end{array}$ \\
\hline 4 votes & & 3 votes \\
\hline 1 (suggests lower value) & 2 (intermediate) & 3 (suggests higher value) \\
\hline $\begin{array}{l}\text { Small health loss without this treatment as measured by } \\
\text { proportional QALY shortfall }\end{array}$ & \multirow[b]{2}{*}{7 votes } & $\begin{array}{l}\text { Substantial health loss without this treatment as } \\
\text { measured by proportional QALY shortfall }\end{array}$ \\
\hline 1 vote & & 3 votes \\
\hline 1 (suggests lower value) & 2 (intermediate) & 3 (suggests higher value) \\
\hline $\begin{array}{l}\text { Will not have a significant impact on improving return to } \\
\text { work and/or overall productivity vs the comparator }\end{array}$ & \multirow[b]{2}{*}{7 votes } & $\begin{array}{l}\text { Will have a significant impact on improving return to work } \\
\text { and/or overall productivity vs the comparator }\end{array}$ \\
\hline 3 votes & & 1 vote \\
\hline$=$ quality-adjusted life-year. & & \\
\hline
\end{tabular}

\section{TABLE 4 Votes on Other Benefits and Contextual Considerations for Nadofaragene Firadenovec}

\begin{tabular}{|c|c|c|}
\hline 1 (suggests lower value) & 2 (intermediate) & 3 (suggests higher value) \\
\hline $\begin{array}{l}\text { Uncertainty or overly favorable model assumptions } \\
\text { creates significant risk that base-case cost-effectiveness } \\
\text { estimates are too optimistic }\end{array}$ & \multirow[b]{2}{*}{7 votes } & $\begin{array}{l}\text { Uncertainty or overly unfavorable model assumptions } \\
\text { creates significant risk that base-case cost-effectiveness } \\
\text { estimates are too pessimistic }\end{array}$ \\
\hline 2 votes & & 2 votes \\
\hline 1 (suggests lower value) & 2 (intermediate) & 3 (suggests higher value) \\
\hline $\begin{array}{l}\text { Very similar mechanism of action to that of other active } \\
\text { treatments }\end{array}$ & \multirow[b]{2}{*}{3 votes } & $\begin{array}{l}\text { New mechanism of action compared with that of other } \\
\text { active treatments }\end{array}$ \\
\hline 0 votes & & 7 votes \\
\hline 1 (suggests lower value) & 2 (intermediate) & 3 (suggests higher value) \\
\hline $\begin{array}{l}\text { Delivery mechanism or relative complexity of regimen } \\
\text { likely to lead to much lower real-world adherence and } \\
\text { worse outcomes relative to an active comparator than } \\
\text { estimated from clinical trials }\end{array}$ & \multirow[b]{2}{*}{3 votes } & $\begin{array}{l}\text { Delivery mechanism or relative complexity of regimen } \\
\text { likely to lead to much higher real-world adherence and } \\
\text { better outcomes relative to an active comparator than } \\
\text { estimated from clinical trials }\end{array}$ \\
\hline 0 votes & & 7 votes \\
\hline 1 (suggests lower value) & 2 (intermediate) & 3 (suggests higher value) \\
\hline $\begin{array}{l}\text { Will not significantly reduce the negative impact of the } \\
\text { condition on the family and caregiver vs the comparator }\end{array}$ & \multirow[b]{2}{*}{9 votes } & $\begin{array}{l}\text { Will significantly reduce the negative impact of the } \\
\text { condition on the family and caregiver vs the comparator }\end{array}$ \\
\hline 1 vote & & 0 votes \\
\hline
\end{tabular}




\section{TABLE 5 Votes on Other Benefits and Contextual Considerations for Oportuzumab Monataox}

\begin{tabular}{|c|c|c|}
\hline 1 (suggests lower value) & 2 (intermediate) & 3 (suggests higher value) \\
\hline $\begin{array}{l}\text { Uncertainty or overly favorable model assumptions } \\
\text { creates significant risk that base-case cost-effectiveness } \\
\text { estimates are too optimistic }\end{array}$ & \multirow[b]{2}{*}{7 votes } & $\begin{array}{l}\text { Uncertainty or overly unfavorable model assumptions } \\
\text { creates significant risk that base-case cost-effectiveness } \\
\text { estimates are too pessimistic }\end{array}$ \\
\hline 2 votes & & 1 vote \\
\hline 1 (suggests lower value) & 2 (intermediate) & 3 (suggests higher value) \\
\hline $\begin{array}{l}\text { Very similar mechanism of action to that of other active } \\
\text { treatments }\end{array}$ & \multirow[b]{2}{*}{3 votes } & $\begin{array}{l}\text { New mechanism of action compared to that of other } \\
\text { active treatments }\end{array}$ \\
\hline 0 votes & & 7 votes \\
\hline 1 (suggests lower value) & 2 (intermediate) & 3 (suggests higher value) \\
\hline $\begin{array}{l}\text { Delivery mechanism or relative complexity of regimen } \\
\text { likely to lead to much lower real-world adherence and } \\
\text { worse outcomes relative to an active comparator than } \\
\text { estimated from clinical trials }\end{array}$ & \multirow[b]{2}{*}{8 votes } & $\begin{array}{l}\text { Delivery mechanism or relative complexity of regimen } \\
\text { likely to lead to much higher real-world adherence and } \\
\text { better outcomes relative to an active comparator than } \\
\text { estimated from clinical trials }\end{array}$ \\
\hline 0 votes & & 2 votes \\
\hline 1 (suggests lower value) & 2 (intermediate) & 3 (suggests higher value) \\
\hline $\begin{array}{l}\text { Will not significantly reduce the negative impact of the } \\
\text { condition on family and caregivers vs the comparator }\end{array}$ & \multirow[b]{2}{*}{8 votes } & $\begin{array}{l}\text { Will significantly reduce the negative impact of the } \\
\text { condition on family and caregivers vs the comparator }\end{array}$ \\
\hline 2 votes & & 0 votes \\
\hline
\end{tabular}

The ICER health benefit price benchmark (HBPB) is a suggested price range based on cost-effectiveness thresholds at the $\$ 100,000$ and $\$ 150,000$ per QALY or evLYG. The HBPB for nadofaragene firadenovec ranged from $\$ 158,600$ to $\$ 262,000$ per year. The HBPB for oportuzumab monatox ranged from $\$ 92,800$ to $\$ 162,100$ per year. We did not calculate a HBPB for pembrolizumab given that its original indication was not for NMIBC. Full results are available on ICER's website at https://icer.org/assessment/ bladder-cancer-2020.

\section{LIMITATIONS OF THE COST-EFFECTIVENESS MODEL}

As noted earlier, there was considerable uncertainty in the long-term estimates for the effectiveness of nadofaragene firadenovec, oportuzumab monatox, and pembrolizumab, and this uncertainty affects any cost-effectiveness analysis of these agents. The model's base-case assumption of an ineffective $(0 \% \mathrm{CR})$ hypothetical comparator is likely overly conservative, so the base-case results can be viewed as the most optimistic scenario and should therefore be interpreted with caution.

\section{Policy Discussion}

The Midwest CEPAC is one of the independent appraisal committees convened by ICER to engage in the public deliberation of the evidence on clinical and cost-effectiveness of health care interventions. The Midwest CEPAC is composed of medical evidence experts, including practicing clinicians, methodologists, and leaders in patient engagement and advocacy. Their deliberation includes input from clinical experts and patient representatives specific to the condition under review, as well as formal comment from manufacturers and the public. A policy roundtable concludes each meeting during which representatives from insurers and manufacturers join clinical experts and patient representatives to discuss how best to apply the findings of the evidence to clinical practice, insurance coverage, and pricing negotiations.

The ICER report on treatments for BCG-unresponsive NMIBC was the subject of a Midwest CEPAC meeting on November 20, 2020. Following the discussion, the Midwest CEPAC panel members deliberated on key questions raised by ICER's report. The results of their votes on the clinical evidence were as follows: (1) the panel voted 7-4 that the clinical evidence was adequate to demonstrate greater net health benefit for nadofaragene firadenovec compared with best supportive care; (2) the panel voted 8-3 that the evidence was adequate to demonstrate greater net health benefit for oportuzumab monatox compared with best supportive care; (3) the panel voted 11-0 that the clinical evidence was inadequate to distinguish the net health 
benefit of nadofaragene firadenovec from oportuzumab monatox; (4) the panel voted 11-0 that the evidence was inadequate to demonstrate a greater net health benefit of nadofaragene firadenovec compared with pembrolizumab; (5) the panel voted 10-1 that the evidence was inadequate to demonstrate a greater net health benefit of oportuzumab monatox compared with pembrolizumab.

The CEPAC panel also voted on "other potential benefits" and "contextual considerations" as part of a process intended to signal to policymakers whether there are important considerations when making judgments about long-term value for money not adequately captured in analyses of clinical and/or cost-effectiveness. Given that pembrolizumab had already been available for more than a year, voting was focused on nadofaragene firadenovec and oportuzumab monatox. The results of these votes are shown in Tables 3-5.

The subsequent policy roundtable discussion explored how best to translate the evidence and additional considerations into clinical practice and into pricing and insurance coverage policies. The full set of policy recommendations can be found in the final evidence report on the ICER website at https://icer.org/assessment/bladder-cancer-2020. Several key policy recommendations follow:

- The FDA should require that manufacturers developing new treatments for BCG-unresponsive NMIBC use randomized trials as the basis of regulatory approval. Manufacturers should also sponsor real-world comparative studies that help evaluate a broad set of patient-relevant outcomes such as quality of life and overall mortality.

- Manufacturers should set prices based on the added clinical value of treatments over lower-cost regimens. Leapfrogging these lower-cost regimens and setting prices relative to higher-cost options adds to the growing financial toxicity of oncology care.

- It seems likely that the FDA label for the emerging treatments will be limited to BCG-unresponsive patients, and payers may therefore wish to consider requiring documentation of a trial of BCG as a criterion for coverage.

- Patient groups should continue their efforts to encourage innovation while pushing life science companies to generate better evidence to guide patient and clinician decision making. Patient groups should embrace their power to speak explicitly about the impact of the high costs of treatments for BCG-unresponsive NMIBC.

- Clinician specialty societies should rapidly move to update guideline recommendations to address the role of these new treatment options.

\section{DISCLOSURES}

Funding for this summary was contributed by Arnold Ventures, California Health Care Foundation, The Donaghue Foundation, Harvard Pilgrim Health Care, and Kaiser Foundation Health Plan to the Institute for Clinical and Economic Review (ICER), an independent organization that evaluates the evidence on the value of health care interventions. ICER's annual policy summit is supported by dues from AbbVie, Aetna, America's Health Insurance Plans, Anthem, Alnylam, AstraZeneca, Biogen, Blue Shield of CA, Boehringer-Ingelheim, Cambia Health Services, CVS, Editas, Evolve Pharmacy, Express Scripts, Genentech/Roche, GlaxoSmithKline, Harvard Pilgrim, Health Care Service Corporation, HealthFirst, Health Partners, Humana, Johnson \& Johnson (Janssen), Kaiser Permanente, LEO Pharma, Mallinckrodt, Merck, Novartis, National Pharmaceutical Council, Pfizer, Premera, Prime Therapeutics, Regeneron, Sanofi, Spark Therapeutics, uniQure, and United Healthcare.
Beinfeld, McKenna, Rind, and Pearson are employed by ICER. Touchette received funding from ICER for work on this report and has also received fees from Monument Analytics and AstraZeneca, unrelated to this work. The University of Illinois at Chicago (UIC) and Touchette hold a patent for the model described in this report. The model is included in ICER's Interactive Modeler, for which a fee is paid to UIC and Touchette. Atlas also received funding from ICER for work on this report.

\section{ACKNOWLEDGMENTS}

The authors thank Maggie O'Grady, Mrinmayee Joshi, Kanya Shah, Rick Chapman, and Monica Fredrick for their contributions to this report.

\section{REFERENCES}

1. Siegel RL, Miller KD, Goding Sauer A, et al. Colorectal cancer statistics, 2020. CA Cancer J Clin. 2020;70(3):145-64.

2. National Cancer Institute. Cancer stat facts: bladder cancer. 2021. Accessed May 17, 2021. https://seer.cancer.gov/statfacts/html/urinb.html

3. Kirkali Z, Chan T, Manoharan M, et al. Bladder cancer: epidemiology, staging and grading, and diagnosis. Urology. 2005;66 (6 Suppl 1):4-34.

4. Yeung C, Dinh T, Lee J. The health economics of bladder cancer: an updated review of the published literature. PharmacoEconomics. 2014;32(11):1093-104.

5. Davies BJ, Hwang TJ, Kesselheim AS. Ensuring access to injectable generic drugs - the case of intravesical BCG for bladder cancer. $\mathrm{N}$ Engl J Med. 2017;376(15):1401-03.

6. Brisbane WG, Holt SK, Winters BR, et al Nonmuscle invasive bladder cancer influences physical health related quality of life and urinary incontinence. Urology. 2019;125:146-53.

7. Winters BR, Wright JL, Holt SK, Dash A, Gore JL, Schade GR. Health related quality of life following radical cystectomy: comparative analysis from the Medicare Health Outcomes Survey. J Urol. 2018;199(3):669-75. 
8. Packiam VT, Johnson SC, Steinberg GD. Non-muscle-invasive bladder cancer: intravesical treatments beyond Bacille Calmette-Guérin. Cancer. 2017;123(3):390-400.

9. Hahn NM, Necchi A, Loriot Y, et al. Role of checkpoint inhibition in localized bladder cancer. Eur Urol Oncol. 2018;1(3):190-98.

10. Flaig T, Spiess P, Agarwal N, et al. Bladder cancer, version 5.2020, NCCN Clinical Practice Guidelines in Oncology. J Natl Compr Canc Netw. 2020;18(3): 329-54.

11. Duplisea JJ, Mokkapati S, Plote D, et al. The development of interferon-based gene therapy for BCG unresponsive bladder cancer: from bench to bedside. World J Urol. 2019;37(10):2041-49.
12. Benedict WF, Tao Z, Kim C-S, et al. Intravesical Ad-IFNalpha causes marked regression of human bladder cancer growing orthotopically in nude mice and overcomes resistance to IFN-alpha protein. Mol Ther. 2004;10(3):525-32.

13. Vlachostergios PJ, Jakubowski CD, Niaz MJ, et al. Antibody-drug conjugates in bladder cancer. Bladder Cancer. 2018;4(3):247-59.

14. Boorjian SA, Alemozaffar M, Konety BR, et al. Intravesical nadofaragene firadenovec gene therapy for BCG-unresponsive non-muscle-invasive bladder cancer: a single-arm, open-label, repeat-dose clinical trial. Lancet Oncol. 2021;22(1):107-17.

15. Boorjian SA, Dinney CPN. Safety and efficacy of intravesical nadofaragene firadenovec for patients with high-grade, BCG unresponsive nonmuscle invasive bladder cancer (NMIBC): Results from a phase III trial. Journal of Clinical Oncology. 2020;38(6).
16. Kapadia E. Clinical development and summary of KEYNOTE-057 efficacy and safety. Merck slide presentation. 2019. Accessed May 6, 2021. https://www. urotoday.com/images/video-slides/ KN057-Efficacy-Safety-17DEC2019-FINAL. pdf

17. Cox E, Saramago P, Kelly J, et al. Effects of bladder cancer on UK healthcare costs and patient health-related quality of life: evidence from the BOXIT Trial. Clin Genitourin Cancer. 2020;18(4):e418-e442.

18. Slater RL, Lai Y, Zhong Y, et al. The cost effectiveness of pembrolizumab versus chemotherapy or atezolizumab as second-line therapy for advanced urothelial carcinoma in the United States. J Med Econ. 2020;23(9):967-77.

19. Kulkarni GS, Finelli A, Fleshner NE, Jewett MA, Lopushinsky SR, Alibhai SM. Optimal management of high-risk T1G3 bladder cancer: a decision analysis. PLoS Med. 2007;4(9):e284. 